Service social

L'approche interculturelle auprès de réfugiés et de nouveaux immigrants, par M. Chiasson-Lavoie, N. Théorêt, M. Gagnon, M. Cantin, J.J. Doricent, G. Azevedo et G. Roy, Montréal, Centre de services sociaux du Montréal métropolitain, 1992.

\title{
François Larose
}

Volume 42, numéro 1, 1993

Culture et intervention

URI : https://id.erudit.org/iderudit/706606ar

DOI : https://doi.org/10.7202/706606ar

Aller au sommaire du numéro

Éditeur(s)

École de service social de l'Université Laval

ISSN

1708-1734 (numérique)

Découvrir la revue

Citer ce compte rendu

Larose, F. (1993). Compte rendu de [L'approche interculturelle auprès de réfugiés et de nouveaux immigrants, par M. Chiasson-Lavoie, N. Théorêt, M. Gagnon, M. Cantin, J.J. Doricent, G. Azevedo et G. Roy, Montréal, Centre de services sociaux du Montréal métropolitain, 1992.] Service social, 42(1),

154-157. https://doi.org/10.7202/706606ar d'utilisation que vous pouvez consulter en ligne. 
Les instruments qui ont servi à la collecte des données sont fournis en annexe ainsi que d'autres outils facilitant la réflexion sur l'intervention interculturelle et féministe.

Les auteures ont voulu ici explorer la problématique de l'interculturel en intervention, afin de pouvoir articuler davantage l'intervention et l'enseignement de ce type d'intervention. La démarche est intéressante et, bien qu'il soit limité quant à l'échantillon d'intervenantes et de clientes, nous avons ici un portrait des diverses situations d'interethnicité intervenante-cliente. Les illustrations, à l'aide de cas types, mettent clairement en évidence ce qu'est le « choc culturel».

Mon malaise se situe davantage sur le plan méthodologique. Nous trouvons, d'une part, une analyse exploratoire quantitative et, d'autre part, une analyse de contenu qui s'inspire, semble-t-il, de la technique des incidents critiques. Malheureusement, cette section est moins claire (p. 60-61) et I'on trouve peu de précisions quant à la méthode réellement utilisée. De la même façon, sur le plan de l'analyse, l'accent est mis sur la fréquence des différentes catégories d'incompréhensions interculturelles, plutôt que sur le sens et l'interprétation à donner. Bref, il semble y avoir là un matériel qualitatif très riche, qui aurait pu donner lieu à un autre type d'analyse. La section 5.6 sur I'intervention féministe, pourtant très importante dans le contexte de cette étude, est brève (neuf pages), ce qui est dommage.

Malgré cette limite, j'estime ce document très pertinent et très utile pour faire avancer la réflexion des intervenantes et des intervenants actuels et futurs en intervention interculturelle. Les auteures elles-mêmes avouent ne pas avoir épuisé leur matériel. J'ose croire qu'il y aura des suites et que la réflexion n'en sera qu'enrichie.

Francine OUELLET

École de service social

Université Laval

\section{L'APPROCHE INTERCULTURELLE AUPRÈS DE RÉFUGIÉS ET DE NOUVEAUX IMMIGRANTS}

M. Chiasson-Lavoie, N. Théorêt, M. Gagnon, M. Cantin, J.J. Doricent, G. Azevedo et G. Roy,

Montréal, Centre de services sociaux du Montréal métropolitain, 1992.

L'ouvrage dont nous traitons ici est le produit d'un collectif d'intervenantes et d'intervenants à l'emploi du CSSMM. Comme le document a une visée didactique, nous y trouvons d'entrée de jeu un glossaire des termes techni- 
ques utilisés. Apparaît ensuite une description du mandat du Service aux migrants et aux immigrants du CSSMM, de même qu'une description des clientèles cibles et une définition des statuts de réfugié ou de migrant à statut précaire. Suit une description, fort judicieuse, des principales sous-catégories de bénéficiaires ainsi que quelques données générales concernant les principales vagues de migration survenues depuis les années 70.

D'une façon générale, la seconde partie du document tente de favoriser une perception holiste de la situation du migrant à statut précaire ou du réfugié. On place le lecteur devant la nécessité de tenir compte de l'interaction de plusieurs facteurs allant de la perception que le migrant peut avoir du service social à la prise de conscience de la valeur des indications que le statut même de l'immigrant nous fournit. On nous présente, dans cette partie de I'ouvrage, une tentative de définir ce qu'est la culture et ce que peut représenter le «choc culturel », c'est-à-dire le processus fort insécurisant par lequel le migrant doit constater les limites d'utilité de ses systèmes de référence par rapport à l'évaluation et à l'analyse de la nouvelle réalité que cette personne doit affronter. Cette prise de conscience d'une certaine «mésadaptation » de ses systèmes de référence concerne tant le rapport à l'environnement physique (ex. : inadaptation au climat, aux systèmes de repère spatiaux utilisés dans un environnement urbain nord-américain, etc.) qu'à l'environnement social (ex.: sentiment d'isolement par rapport aux réseaux familiaux, différences de systèmes de valeurs, etc.).

La troisième ainsi que la quatrième partie du document tentent de déterminer certains paramètres pouvant se révéler utiles en tant que guide pour l'action à l'intention de la praticienne ou du praticien social désirant adapter la perspective de son intervention à la réalité interculturelle. On y favorise la prise de conscience de sa propre identité sociale et culturelle et I'identification de son rapport à un cadre institutionnel qui est, lui-même, un produit marqué culturellement. On y favorise aussi le développement d'une dynamique à l'intérieur de laquelle la prise de conscience des éléments formant I'identité de l'intervenant et celle du client permet l'acceptation des différences, notamment sur le plan des attentes et des modalités de la communication, et favorise, par ce moyen, le développement d'une relation d'aide efficace.

Dans la perspective privilégiée par les auteurs du document, la prise de conscience des «marqueurs» de l'identité du praticien et de celle du client forme un processus dialectique qui, seul, peut être garant de l'acquisition $d^{\prime}$ 'attitudes et de valeurs pertinentes dans I'approche interculturelle. Ce processus de conscientisation permettra, par ailleurs, à la praticienne ou au praticien social de se démarquer des institutions aux yeux de la clientèle et, en conséquence, améliorera les chances que le travail d’information que l'intervenant doit effectuer s'avère efficace.

Le document se termine par l'énoncé des caractéristiques et des services mis en place par le CSSMM afin de favoriser le développement d'une perspective interculturelle dans la pratique de ses membres. Outre l'adapta- 
tion du contexte organisationnel (multiethnicité du personnel, concertation avec les organismes «ethniques» du milieu, disponibilité d'une banque $d^{\prime}$ interprètes, etc.), c'est la perspective dans laquelle s'effectue la livraison des services qui est affectée. L'approche interculturelle signifiera diverses choses selon que l'on se place dans une perspective de court, de moyen ou de long terme. Le court terme est souvent marqué par une situation de «crise» où le migrant à statut précaire et le réfugié doivent, avant tout, être pourvus du minimum permettant leur survie physique et le maintien d'un minimum d'équilibre psychique. Le moyen terme permet l'ouverture de la perspective d'adaptation et d'ajustement de la relation praticien-client à travers le processus d'auto et d'interconscientisation des systèmes gérant les rapports à autrui. Le long terme, enfin, implique la baisse de l'importance de l'intervention de la praticienne ou du praticien et l'apprentissage de l'utilisation autonome, par le migrant, des ressources du milieu permettant son insertion et son intégration sociale et économique dans le respect de ce qui caractérise son identité et l'identité des citoyens « de souche».

Le document est complété par trois annexes qui, à elles seules, en justifieraient la diffusion. On y trouvera, dans un premier temps, une grille descriptive d'éléments clés caractérisant le profil type de catégories de clientèles migrantes ou réfugiées, la définition des aspects saillants de la problématique et du type de services qui doivent être livrés d'urgence à ces clientèles.

La seconde annexe consiste en une traduction-synthèse d'un ouvrage portant sur diverses caractérisques des principales approches en service social auprès des clientèles « ethniques » aux États-Unis. Sa consultation permet, en un bref survol, de prendre conscience du caractère idéologiquement marqué des ces approches. Elle permet aussi au lecteur de voir que, si elles présentent toutes des aspects de congruence avec les besoins des clientèles, elles présentent aussi des lacunes et que, dans certains cas, leur application peut se révéler plus nuisible qu'autrement auprès de certaines clientèles ethniques.

La dernière annexe consiste en un hybride de liste de contrôle et de grille d'autoquestionnement devant guider la pratique «consciente» de l'intervenant social auprès de clientèles d'origines ethniques diverses. Elle ne se veut pas un guide restrictif ou exhaustif, mais un instrument qui permet au praticien de prendre en considération la réalité objective (contexte de la migration, contexte familial, rapport «légal » avec les services de santé et les services sociaux) et la réalité subjective des clientèles cibles.

En bref, d'une façon générale il s'agit d'un document qui pourra s'avérer utile et formateur pour I'intervenante ou l'intervenant affrontant la nouvelle réalité des clientèles multiethniques en situation de crise. Néanmoins, le lecteur devrait «se questionner » et aborder avec circonspection certains des éléments «théoriques » présentés. Deux exemples tirés du glossaire suffiront à illustrer ce propos. Sous la rubrique «stéréotypes » on a droit à une définition simpliste et caricaturale, tenant plus du jugement de valeur que de la définition opératoire. On doit cette définition au coauteur d'un document interne 
produit à l'intérieur du circuit collégial. II existe pourtant des définitions opératoires du stéréotype qui sont, d'emblée, mises en relation avec le concept de «représentation sociale» dont le stéréotype* est le plus souvent un élément structurant. De la même façon, pourquoi nous présenter une définition à la fois partielle et douteuse du concept d'acculturation produite par un glorieux inconnu, alors que la littérature scientifique en anthropologie et en psychologie renvoie généralement à une définition originale produite par Herskovits (1938)?

Dans l'ensemble, il s'agit donc d'un document d'information intéressant qui ne saurait, cependant, être considéré d'aucune façon comme un document de formation.

FrançOis LAROSE

Département d'enseignement au préscolaire et au primaire Université de Sherbrooke

\section{PRATIQUES INTERCULTURELLES SOUS L'ANGLE DE LA MODERNITÉ}

Ghislaine Roy,

Montréal, Centre de services sociaux du Montréal métropolitain, 1991.

Ce document, édité par le CSSMM, est en fait la reproduction d'un mémoire de maîtrise en service social rédigé par une intervenante chevronnée du réseau des services sociaux. L'auteure y définit en premier lieu son cadre théorique. Après avoir situé la pluriethnicité québécoise dans le contexte historique de I'accès à la "modernité », I'auteure aborde rapidement le concept du multiculturalisme en tant que discours idéologiquement marqué. Elle situe ensuite le développement des réseaux formels ainsi que des pratiques des services sociaux québécois à l'intérieur d'une même perspective dynamique d'accès à la modernité. Elle situe notamment, de façon fort judicieuse, la notion de parallélisme des services, des réseaux et des infrastructures entre les deux communautés culturelles dont le statut de pérennité est reconnu: les communautés franco-québécoise et angloquébécoise.

Si la notion de modernité est à la fois bien définie sur le plan théorique et clairement articulée dans une perspective socio-historique, certains construits complexes sont définis quelque peu rapidement et sommairement. Il en est ainsi du construit de culture ou de I'utilisation dans une

* Voir à ce propos: J.-P. Leyens, Sommes-nous tous des psychologues? Bruxelles : Mardaga, 1983. 